\title{
EXACT SOLUTION OF FIN PROBLEM WITH LINEAR TEMPERATURE-DEPENDENT THERMAL CONDUCTIVITY
}

\author{
A.H. Abdel Kader, M.S. Abdel Latif, H.M. Nour \\ Mathematics and Engineering Physics Department, Faculty of Engineering \\ Mansoura University, Egypt \\ Leaderabass87@gmail.com,m_gazia@mans.edu.eg,hanour@mans.edu.eg
}

Received: 20 September 2016; accepted: 15 November 2016

\begin{abstract}
In this paper, we obtain the general exact solution of a nonlinear fin equation which governs heat transfer in a rectangular fin with linear temperature-dependent thermal conductivity using the partial Noether method. The relationship between the fin efficiency and the thermo-geometric fin parameter is obtained. Additionally, we obtained the relationship among the fin effectiveness, the thermo-geometric fin parameter and the Biot number.
\end{abstract}

Keywords: exact solution, fin equation, fin efficiency, thermal conductivity

\section{Introduction}

In this paper, we assume that the rectangular fin subjected to some assumptions such as steady state heat transfer operation with no heat generation, the fin tip is insulated, and the heat transfer is one dimensional. Under these assumptions, the energy balance equation of rectangular fin is given by [1-11]

$$
A_{c} \frac{d}{d X}\left(K(T) \frac{d T}{d X}\right)-P h\left(T-T_{a}\right)=0,
$$

where, $T$ is the fin temperature, $X$ is the axial distance measured from the fin tip, $A_{c}$ is the cross-sectional area of the fin, $P$ is the fin perimeter, $k(T)$ is the thermal conductivity of the fin, $h$ is the heat transfer coefficient and $T_{a}$ is the ambient temperature.

Here, we take the heat transfer coefficient $h$ as a constant and the thermal conductivity $k(T)$ as a linear function of temperature [1-9]

$$
k(T)=K_{a}\left(1+\tau\left(T-T_{a}\right)\right),
$$

where $K_{a}$ is the thermal conductivity of the fin at the ambient temperature $T_{a}, \tau$ is a constant. 
Substituting (2) into (1), we obtain

$$
A_{c} K_{a} \frac{d}{d X}\left(\left(1+\tau\left(T-T_{a}\right)\right) \frac{d T}{d X}\right)-P h\left(T-T_{a}\right)=0
$$

To make Eq. (3) dimensionless; the following transformations are introduced [1-14]

$$
u=\frac{T-T_{a}}{T_{b}-T_{a}}, \quad x=\frac{x}{b}, \quad \beta=\tau\left(T_{b}-T_{a}\right), \quad \psi^{2}=\frac{P h b^{2}}{K_{a} A_{c}},
$$

where, $b$ is the length of fin, $T_{b}$ is the temperature of the heat source where the fin is attached and the parameter $\psi$ is called thermo geometric fin parameter. Using the transformations (4), Eq. (3) becomes

$$
\frac{d}{d x}\left((1+\beta u) \frac{d u}{d x}\right)-\psi^{2} u=0
$$

Equation (5) can be rewritten as

$$
u^{\prime \prime}(x)+\beta u u^{\prime \prime}(x)+\beta u^{\prime}(x)^{2}-\psi^{2} u(x)=0 .
$$

The boundary conditions are:

At the fin tip $(\mathcal{X}=0)$, since the fin tip is insulated, so

$$
\frac{d T}{d X}=0 .
$$

Using the transformation (4), Eq. (7) becomes

$$
u^{\prime}(0)=0 .
$$

At the fin base $(X=b)$, the fin temperature is the same temperature as the heat source $T_{b}$

$$
T(b)=T_{b} .
$$

Using the transformation (4), Eq. (9) becomes

$$
u(1)=1 .
$$

Approximate solutions of Eq. (6) with boundary conditions (8) and (10) are investigated using the Parameterized Perturbation method in [1], by using optimal homotopy asymptotic method in [2], by using the homotopy analysis method in $[3,4]$, by using the Residue minimization technique in [5], by using the variational iteration method in [6] and by using the decomposition method in $[7,8]$. 
The homotopy analysis method is widely used in investigating many fin problems in [12-14]. In this paper, we will obtain the exact solution of Eq. (6) using the partial Noether method.

The paper will be organized as follows: In section 2, the exact solution of Eq. (6) is obtained using the partial Noether method. In section 3, the fin efficiency will be discussed. In section 4, the fin effectiveness is studied. In section 5, we will discuss the obtained results in this paper.

\section{Partial Noether Method}

Definition $[15,16]$. A Lie operator $X$ of a form

$$
X=\xi \frac{\partial}{\partial x}+\eta \frac{\partial}{\partial u}+\left(D_{x} \eta-u^{\prime} D_{x} \xi\right) \frac{\partial}{\partial u^{\prime}}+\cdots
$$

is called a partial Noether operator corresponding to a partial Lagrangian $L$, if there exists a function $B$, such that

$$
X(L)+L D_{x}(\xi)=\left(\eta-u^{\prime} \xi\right) \frac{\delta L}{\delta u}+D_{x}(B)
$$

where $D_{x}$ is the total differentiation with respect to $x$ and $\frac{\delta}{\delta u}$ is called the Euler-Lagrange operator, which are defined as,

$$
\begin{gathered}
D_{x}=\frac{\partial}{\partial x}+u^{\prime} \frac{\partial}{\partial u}+u^{\prime \prime} \frac{\partial}{\partial u^{\prime}}+\cdots \\
\frac{\delta}{\delta u}=\frac{\partial}{\partial u}-D_{x}\left(\frac{\partial}{\partial u^{\prime}}\right)+\cdots
\end{gathered}
$$

Theorem $[15, \mathbf{1 6}]$. If the Lie operator (11) is a partial Noether operator corresponding to a partial Lagrangian $L$ of Eq. (6), then the first integral $I$ of (6) is given by

$$
I=B-N(L),
$$

which is satisfied by the conservation law

$$
\left.D_{x}(I)\right|_{(6)}=0,
$$

where, $N$ is a Noether operator which is defined as:

$$
N=\xi+\left(\eta-u^{\prime} \xi\right) \frac{\delta}{\delta u^{\prime}}+\cdots,
$$


where,

$$
\frac{\delta}{\delta u^{\prime}}=\frac{\partial}{\partial u^{\prime}}-D_{x}\left(\frac{\partial}{\partial u^{\prime \prime}}\right)+\cdots
$$

Consider the partial Lagrangian of Equation (6) $[15,16]$

$$
L=\frac{\left(u^{\prime}\right)^{2}}{2}
$$

where,

$$
\frac{\delta L}{\delta u}=-u^{\prime \prime}=\frac{\beta\left(u^{\prime}\right)^{2}-\psi^{2} u}{1+\beta u}
$$

To obtain the partial Noether operator of Eq. (6), we will substitute (18) and (19) into the condition (12) to obtain

$$
X\left(\frac{\left(u^{\prime}\right)^{2}}{2}\right)+\frac{\left(u^{\prime}\right)^{2}}{2} D_{x}(\xi)=\left(\eta-u^{\prime} \xi\right)\left(\frac{\beta\left(u^{\prime}\right)^{2}-\psi^{2} u}{1+\beta u}\right)+D_{x}(B)
$$

Substituting (11) into (20), we obtain the determining equation

$$
\left(D_{x} \eta-u^{\prime} D_{x} \xi\right) u^{\prime}+\frac{\left(u^{\prime}\right)^{2}}{2} D_{x}(\xi)=\left(\eta-u^{\prime} \xi\right)\left(\frac{\beta\left(u^{\prime}\right)^{2}-\psi^{2} u}{1+\beta u}\right)+D_{x}(B) .
$$

Let, $\xi=\xi(x, u), \eta=\eta(x, u)$ and $B=B(x, u)$, the determining equation (21) becomes

$$
\begin{aligned}
-B_{x}+\frac{\psi^{2} u}{\beta u+1} \eta & +\left(-B_{u}-\frac{\psi^{2} u}{\beta u+1} \xi+\eta_{x}\right) u^{\prime}+\left(\frac{\beta}{\beta u+1} \xi-\frac{1}{2} \xi_{u}\right)\left(u^{\prime}\right)^{3} \\
& +\left(-\frac{\beta \eta}{\beta u+1}+\eta_{u}-\frac{1}{2} \xi_{x}\right)\left(u^{\prime}\right)^{2}=0
\end{aligned}
$$

Equating the coefficients of the derivatives of $u$ with zero, we obtain

$$
\begin{array}{lc}
\left(u^{\prime}\right)^{3}: & \frac{\beta}{\beta u+1} \xi-\frac{1}{2} \xi_{u}=0, \\
\left(u^{\prime}\right)^{2}: & -\frac{\beta \eta}{\beta u+1}+\eta_{u}-\frac{1}{2} \xi_{x}=0, \\
\left(u^{\prime}\right): & -B_{u}-\frac{\psi^{2} u}{\beta u+1} \xi+\eta_{x}=0, \\
\left(u^{\prime}\right)^{0}: & -B_{x}+\frac{\psi^{2} u}{\beta u+1} \eta=0 .
\end{array}
$$


The solution of system (23)-(26) is given by

$$
\xi=6(1+\beta u)^{2}, \quad \eta=0, \quad B=-u^{2}(3+2 \beta u) \psi^{2} .
$$

Substituting (18) into (16), we obtain

$$
N(L)=\eta u^{\prime}-\xi \frac{\left(u^{\prime}\right)^{2}}{2} .
$$

Substituting (27) and (28) into (14), we obtain

$$
I=-u^{2}(3+2 \beta u) \psi^{2}+3(1+\beta u)^{2}\left(u^{\prime}\right)^{2} .
$$

Suppose the first integral $I=c_{1}$, hence, we obtain

$$
-u^{2}(3+2 \beta u) \psi^{2}+3(1+\beta u)^{2}\left(u^{\prime}\right)^{2}=c_{1},
$$

where $c_{1}$ is a constant. Using the boundary condition (8), we can determine the constant $c_{1}$ as follows

$$
-u_{0}^{2}\left(3+2 \beta u_{0}\right) \psi^{2}=c_{1}
$$

where, $u_{0}$ is the temperature of fin at the fin tip $(x=0)$. Substituting (31) into (30), we obtain

$$
u_{0}^{2}\left(3+2 \beta u_{0}\right) \psi^{2}-u^{2}(3+2 \beta u) \psi^{2}+3(1+\beta u)^{2}\left(u^{\prime}\right)^{2}=0,
$$

Let,

$$
u=g(x)-\frac{1}{\beta}
$$

Hence, Eq. (32) becomes

$$
g^{\prime}(x)^{2}=\frac{\psi^{2}}{3 \beta^{4} g(x)^{2}}\left(-\left(\beta u_{0}+1\right)^{2}\left(2 \beta u_{0}-1\right)+2 \beta^{3} g(x)^{3}-3 \beta^{2} g(x)^{2}\right) \text {. }
$$

Integrating Eq. (33), we obtain

$$
\int_{0}^{1} d x=\frac{-\sqrt{3} \beta^{2}}{\psi} \int_{u_{0}+\frac{1}{\beta}}^{u+\frac{1}{\beta}} g\left(\left(-\left(\beta u_{0}+1\right)^{2}\left(2 \beta u_{0}-1\right)+2 \beta^{3} g^{3}-3 \beta^{2} g^{2}\right)\right)^{-\frac{1}{2}} d g .
$$

Hence, we obtain the following exact implicit solution of Eq. (5) 


$$
\begin{aligned}
& x=-\frac{\sqrt{3}}{\sqrt{-2 \gamma} \psi}\left(\gamma \boldsymbol{E}\left(\sin ^{-1}\left(\frac{1}{\sqrt{2} \sqrt[4]{3}} \sqrt{\rho-\frac{4 \beta}{\alpha} u_{0}}\right) \mid \frac{2 \sqrt{3} \alpha}{\gamma}\right)\right. \\
& -\gamma \boldsymbol{E}\left(\sin ^{-1}\left(\frac{1}{\sqrt{2} \sqrt[4]{3}} \sqrt{\rho-\frac{4 \beta}{\alpha} u}\right) \mid \frac{2 \sqrt{3} \alpha}{\gamma}\right) \\
& +4\left(\beta u_{0}+1\right)\left(\boldsymbol{F}\left(\sin ^{-1}\left(\frac{1}{\sqrt{2} \sqrt[4]{3}} \sqrt{\rho-\frac{4 \beta}{\alpha} u_{0}}\right) \mid \frac{2 \sqrt{3} \alpha}{\gamma}\right)\right. \\
& \left.\left.-\boldsymbol{F}\left(\sin ^{-1}\left(\frac{1}{\sqrt{2} \sqrt[4]{3}} \sqrt{\rho-\frac{4 \beta}{\alpha}} u\right) \mid \frac{2 \sqrt{3} \alpha}{\gamma}\right)\right)\right)
\end{aligned}
$$

where, $\boldsymbol{E}$ is the incomplete elliptic integral of the second kind, which is defined as [17]

$$
\boldsymbol{E}(\phi \mid m)=\int_{0}^{\phi} \sqrt{1-m \sin ^{2}(\theta)} \mathrm{d} \theta,
$$

$\boldsymbol{F}$ is the incomplete elliptic integral of the first kind, which is defined as [17]

$$
\boldsymbol{F}(\phi \mid m)=\int_{0}^{\phi} \frac{1}{\sqrt{1-m \sin ^{2}(\theta)}} \mathrm{d} \theta
$$

and $\alpha=\sqrt{3-4 \beta u_{0}-4 \beta^{2} u_{0}^{2}}, \gamma=-3+\sqrt{3} \alpha-6 \beta u_{0}, \rho=\sqrt{3}-\frac{1}{\alpha}\left(3+2 u_{0} \beta\right)$. The solution (35) has an unknown parameter namely $u_{0}$. This parameter can be easily determined with the help of the boundary condition $u(1)=1$ as follows:

$$
\begin{aligned}
1=-\frac{\sqrt{3}}{\sqrt{-2 \gamma} \psi} & \gamma \boldsymbol{E}\left(\sin ^{-1}\left(\frac{1}{\sqrt{2} \sqrt[4]{3}} \sqrt{\rho-\frac{4 \beta}{\alpha} u_{0}}\right) \mid \frac{2 \sqrt{3} \alpha}{\gamma}\right) \\
& -\gamma \boldsymbol{E}\left(\sin ^{-1}\left(\frac{1}{\sqrt{2} \sqrt[4]{3}} \sqrt{\rho-\frac{4 \beta}{\alpha}}\right) \mid \frac{2 \sqrt{3} \alpha}{\gamma}\right) \\
& +4\left(\beta u_{0}+1\right)\left(\boldsymbol{F}\left(\sin ^{-1}\left(\frac{1}{\sqrt{2} \sqrt[4]{3}} \sqrt{\rho-\frac{4 \beta}{\alpha} u_{0}}\right) \mid \frac{2 \sqrt{3} \alpha}{\gamma}\right)\right. \\
& \left.\left.-\boldsymbol{F}\left(\sin ^{-1}\left(\frac{1}{\sqrt{2} \sqrt[4]{3}} \sqrt{\rho-\frac{4 \beta}{\alpha}}\right) \mid \frac{2 \sqrt{3} \alpha}{\gamma}\right)\right)\right)
\end{aligned}
$$


Equation (36) shows the relation between the temperature at fin tip $u_{0}$ and the thermo-geometric parameter $\psi$ and $\beta$.

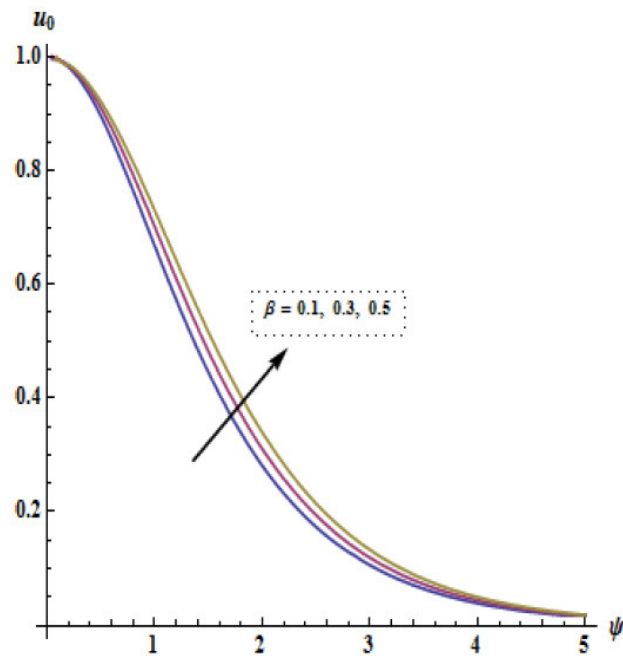

Fig. 1. Plot of the relation (36) between $\psi$ and $u_{0}$ for various values of $\beta$

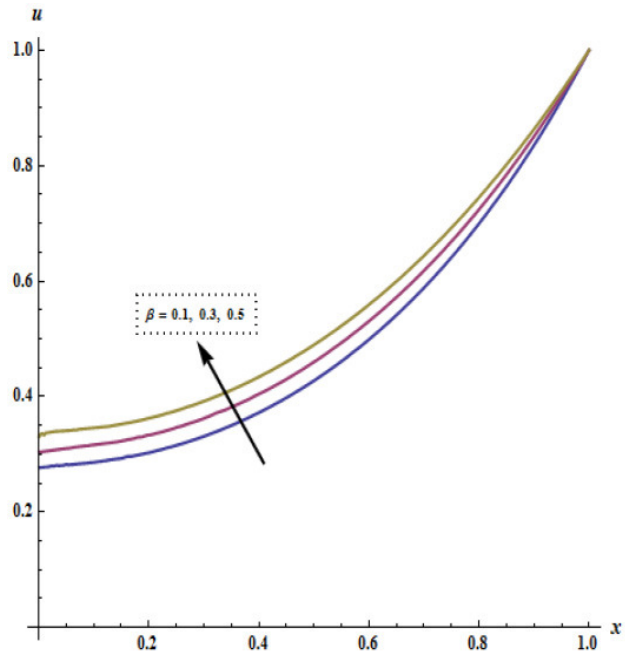

Fig. 2. Plot of the relation (35) between $u$ and $x$ for various values of $\beta$ when $\psi=2$

Figure 1 shows the effect of the thermo-geometric parameter $\psi$ on the fin tip temperature $u_{0}$. We find that the fin tip temperature $u_{0}$ decreases with increasing $\psi$. Figure 2 shows the distribution of fin temperature $u$ along the fin. We find that the fin temperature decreases with increasing $x$.

\section{Fin efficiency}

The fin efficiency $\boldsymbol{\eta}$ is the ratio of the actual heat transfer rate $Q_{a}$ from the fin to ideal heat transfer $Q_{i}$ rate from the fin if the entire fin were at base temperature [3-11]

$$
\boldsymbol{\eta}=\frac{Q_{a}}{Q_{i}}=\frac{\int_{0}^{b} p h\left(T-T_{a}\right) d X}{p b h\left(T_{b}-T_{a}\right)}=\int_{0}^{1} u d x
$$

Using Eq. (5), Eq. (37) becomes

$$
\boldsymbol{\eta}=\frac{1}{\psi^{2}} \int_{0}^{1} \frac{d}{d x}\left((1+\beta u) \frac{d u}{d x}\right) d x=\frac{1}{\psi^{2}}\left((1+\beta u(1)) u^{\prime}(1)-(1+\beta u(0)) u^{\prime}(0)\right)
$$

Using the boundary conditions (8) and (10), Eq. (38) becomes

$$
\boldsymbol{\eta}=\frac{(1+\beta)}{\psi^{2}} u^{\prime}(1)
$$


From (32), when $x=1$, we obtain

$$
u^{\prime}(1)=\frac{\psi}{\sqrt{3}(1+\beta)} \sqrt{(3+2 \beta)-u_{0}^{2}\left(3+2 \beta u_{0}\right)} .
$$

Substituting (40) into (39), we obtain

$$
\boldsymbol{\eta}=\frac{1}{\sqrt{3} \psi} \sqrt{(3+2 \beta)-u_{0}^{2}\left(3+2 \beta u_{0}\right)} .
$$

Using the relations (41) and (36), we can plot the relation between the efficiency $\boldsymbol{\eta}$ and the thermo-geometric fin parameter $\psi$ (see Figure 3).

\section{Fin effectiveness}

Fin effectiveness $\epsilon$ is the ratio of heat transferred from the fin area $Q_{a}$ to the heat which would be transferred if entire fin area was at base temperature $Q_{b}$ [11]

$$
\epsilon=\frac{Q_{a}}{Q_{b}}=\frac{\int_{0}^{b} p h\left(T-T_{a}\right) d x}{A_{c} h\left(T_{b}-T_{a}\right)}=G \int_{0}^{1} u d x .
$$

where, $G=\frac{P b}{A_{C}}$ is a parameter which depends on the fin geometry.

From (37), we find

$$
\epsilon=G \boldsymbol{\eta}
$$

Substituting (41) into (43), we obtain

$$
\epsilon=\frac{G}{\sqrt{3} \psi} \sqrt{(3+2 \beta)-u_{0}^{2}\left(3+2 \beta u_{0}\right)} .
$$

The parameter $\psi$ can be rewritten in the form

$$
\psi^{2}=B i G \text {. }
$$

where, $B i=\frac{h b}{K_{a}}$ is the Biot number.

Substituting (45) into (44), we obtain

$$
\epsilon=\sqrt{\frac{G}{3 B i}\left((3+2 \beta)-u_{0}^{2}\left(3+2 \beta u_{0}\right)\right)} .
$$

Using the relations (45), (46) and (36), we can plot the relation between the fin effectiveness $\epsilon$ and the parameters $G$ and $B i$ (Figures 4 and 5). 


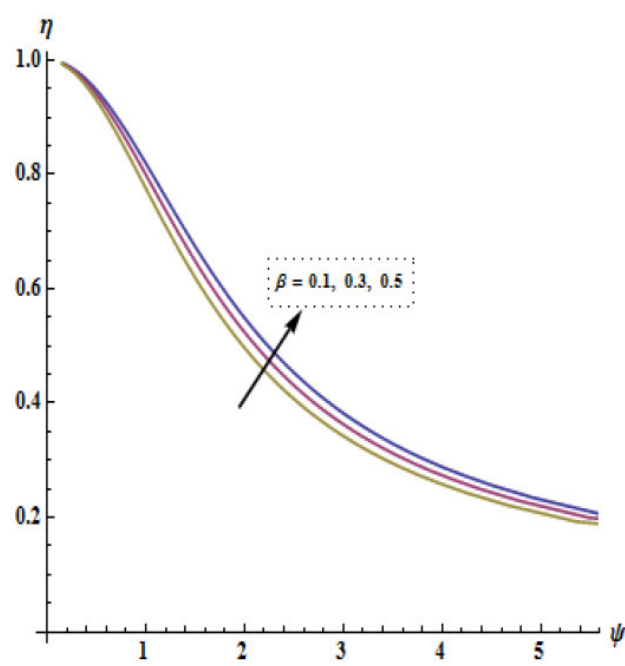

Fig. 3. Plot the relation between the efficiency $\eta$ and the thermo-geometric parameter $\psi$ for various values of $\beta$

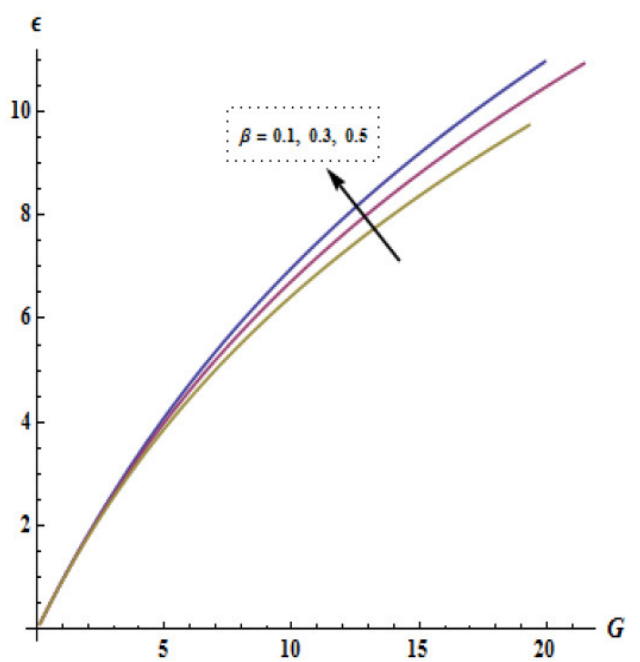

Fig. 4. Plot the relation between the effectiveness of fin $\epsilon$ and $G$ when $B i=0.2$ for various values of $\beta$

Figure 3 shows the effect of the thermo-geometric parameter $\psi$ on fin efficiency $\eta$. We find that the fin efficiency $\eta$ decreases with increasing $\psi$. Figure 4 shows the effect of the parameter $G$ on fin effectiveness $\epsilon$. We find that fin effectiveness $\epsilon$ increases with increasing $G$.

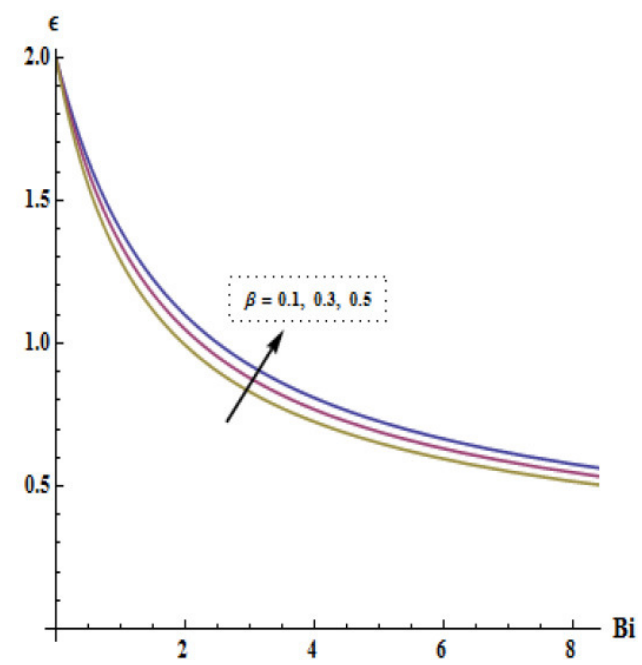

Fig. 5. Plot the relation between the fin effectiveness $\epsilon$ and $B i$ when $G=2$ for various values of $\beta$

Figure 5 shows the effect of the Biot number on fin effectiveness $\epsilon$. We find that fin effectiveness $\epsilon$ decreases with increasing $B i$. 


\section{Discussions and concluding remarks}

In this paper, we obtain the general exact solution (35) of the fin equation (5) which is subjected to the boundary conditions (8) and (10). The solution is valid for all values of the thermo-geometric fin parameters $\psi$ and $\beta$. We observe in Figure 1 that the fin tip temperature $u_{0}$ decreases with an increase in the thermo-geometric parameter $\psi$. Figure 2 shows that the fin temperature $u$ increases with an increasing $x$ (in other words, the temperature increases when approaching a heat source). The relation between the fin efficiency $\eta$ and the parameters $\psi$ and $\beta$ is obtained. Figure 3 shows that the fin efficiency $\eta$ decreases with increasing the thermo-geometric parameter $\psi$. The relation between the fin effectiveness $\epsilon$ and the parameters, $\beta$ and Biot number $B i$ is obtained. Figures 4 and 5 show that the fin effectiveness $\epsilon$ increases when increasing $G$ and decreases when increasing the Biot number $B i$.

\section{References}

[1] Seiyed Ghasemi E., Ali Zolfagharian, M. Hatam, Ganji D.D., Analytical thermal study on nonlinear fundamental heat transfer cases using a novel computational technique, Applied Thermal Engineering 2016, 98, 88-97.

[2] Saeed Dinarvand, Reza Hosseini, Optimal homotopy asymptotic method for convectiveradiative cooling of a lumped system, and convective straight fin with temperature-dependent thermal conductivity, Afr. Mat. 2013, 24, 103-116.

[3] Srikumar Panda, A study on nonlinear wet fin problem using homotopy analysis method, Int. J. Appl. Comput. Math, 2016, DOI: 10.1007/s40819-016-0188-1.

[4] Mustafa Inc: Application of homotopy analysis method for fin efficiency of convective straight fins with temperature-dependent thermal conductivity, Mathematics and Computers in Simulation 2008, 79, 189-200.

[5] Kulkarni D.B., Joglekar M.M., Residue minimization technique to analyze the efficiency of convective straight fins having temperature-dependent thermal conductivity, Applied Mathematics and Computation 2009, 215, 2184-2191.

[6] Safa Bozkurt Coskun, Mehmet Tarik Atay, Fin efficiency analysis of convective straight fins with temperature dependent thermal conductivity using variational iteration method, Applied Thermal Engineering 2008, 28, 2345-2352.

[7] Cihat Arslanturk, A decomposition method for fin efficiency of convective straight fins with temperature-dependent thermal conductivity, International Communications in Heat and Mass Transfer 2005, 32, 831-841.

[8] Ching-Huang Chiu, Cha'o-Kuang Chen, A decomposition method for solving the convective longitudinal fins with variable thermal conductivity, Int. J. Heat and Mass Transfer. 2002, 45, 2067-2075.

[9] Abdel Latif M.S., Abdel Kader A.H., Nour H.M., Exact implicit solution of nonlinear heat transfer in rectangular straight fin using symmetry reduction methods, Appl. Appl. Math. 2015, 10, 2, 864-877.

[10] Abdel Kader A.H., Abdel Latif M.S., Nour H.M., General exact solution of the fin problem with variable thermal conductivity, Propulsion and Power Research 2016, 5(1), 63-69.

[11] Abdel Kader A.H., Abdel Latif M.S., Nour H.M., General exact solution of the fin problem with the power law temperature - dependent thermal conductivity, Math. Meth. Appl. Sci. 2016, $39,1513-1521$. 
[12] Srikumar Panda, Arka Bhowmik, Ranjan Das, Ramjee Repaka, Subash Martha C., Application of homotopy analysis method and inverse solution of a rectangular wet fin, Energy Conversion and Management 2014, 80, 305-318.

[13] Arka Bhowmik, Srikumar Panda, Ranjan Das, Ramjee Repaka, Subash Marthaet C., Inverse analysis of conductive-convective wet triangular fin for predicting thermal properties and fin dimensions, Inverse Problems in Science and Engineering 2014, 22(8), 1367-1393.

[14] Srikumar Panda, Arka Bhowmik, Homotopy analysis method for thermal analysis of wet fin with all nonlinearity, International Conference on Engineering (NUiCONE), 2013, 1-6, DOI:10.1109/NUiCONE.2013. 6780197.

[15] Kara A.H., Mahomed F.M., Naeem I., Wafo Soh C., Partial Noether operators and first integrals via partial Lagrangians, Math. Meth. Appl. Sci. 2007, 30, 2079-2089.

[16] Naeem I., Mahomed F.M., Noether, partial Noether operators and first integrals for a linear system, J. Math. Anal. Appl. 2008, 342, 70-82.

[17] Frank Olver W.J., Lozier D.W., Boisvert R.F., Clark C.W., Nist Handbook of Mathematical Functions 2010. 